\title{
EchoGéo
}

$13 \mid 2010$

Afrique, 50 ans d'indépendance : État et territoires

\section{Panorama de revues de géographie africaines consultables en France}

\section{Bernadette Joseph}

\section{OpenEdition}

1 Journals

Édition électronique

URL : https://journals.openedition.org/echogeo/12050

DOI : $10.4000 /$ echogeo. 12050

ISSN : 1963-1197

\section{Éditeur}

Pôle de recherche pour l'organisation et la diffusion de l'information géographique (CNRS UMR 8586)

Référence électronique

Bernadette Joseph, "Panorama de revues de géographie africaines consultables en France », EchoGéo [En ligne], 13 | 2010, mis en ligne le 20 septembre 2010, consulté le 03 août 2021. URL: http://journals.openedition.org/echogeo/12050 ; DOI : https://doi.org/10.4000/echogeo.12050

Ce document a été généré automatiquement le 3 août 2021.

EchoGéo est mis à disposition selon les termes de la licence Creative Commons Attribution - Pas d'Utilisation Commerciale - Pas de Modification 4.0 International (CC BY-NC-ND) 


\title{
Panorama de revues de géographie africaines consultables en France
}

\author{
Bernadette Joseph
}

\section{Introduction}

1 Cette recension porte sur les revues de géographie, publiées par les universités africaines, depuis les années 1960. Les périodiques multidisciplinaires concernant l'Afrique, édités par des organismes de recherches français en sont exclus.

2 Ce panorama, a été réalisé par l'interrogation systématique des sites des universités africaines, du SUDOC, de BNF- OPALE, des catalogues de bibliothèques hors réseaux. Il se veut le plus exhaustif possible actuellement, mais il faut tenir compte des variations fréquentes de la production éditoriale dans de domaine des périodiques et de la pérennité parfois aléatoire des sites web.

\section{Revues Imprimées}

\section{Afrique du sud}

Acta geographica.- Cape Town : Balkema, 1967 (1 vol.). ISSN 0065-1230.

4 South african journal.- Johannesburg: South African Geographical Society, 1929- . Bisannuel. ISSN : 0373-6245 (full text on line, Current issue, vol. 91, 2, 2009).

5 Suid-afrikaanse geograaf = South african geography.- Stellen Bosch: Verenigning vir geografie, 1972- . Semestriel (suite de Tydskrif vir aardryskunde). ISSN : 0021-8243.

\section{Algérie}

6 Annales Algériennes de géographie.- Alger : Université d'Alger, Institut de géographie, 1966-1972. Semestriel. ISSN : 0570-1546. 
7 Bulletin de la Société de Géographie et d'archéologie d'Oran.- Oran: Société de géographie et d'archéologie, 1966-1983. Irrégulier. ISSN: 1111-1577. (devient Bulletin de la Société de géographie et d'archéologie de la province d'Oran ); (devient Bulletin trimestriel de la Société de Géographie et d'archéologie d'Oran, 1982-1984. ISSN: 1015-1125).

8 Bulletin des sciences géographiques.- Alger: Institut national de cartographie et télédétection, 1998-. Semestriel. ISSN: 1112-2293 (suite de Revue des sciences géographiques).

Cahiers géographiques de l'ouest.- Oran: Université d'Oran, 1978- . Irrégulier (reçu dans les Bibliothèques françaises entre 1978-1987).

Insaniyat: revue algérienne d'anthropologie et des sciences sociales.- Oran : CRASC, 1997- . Irrégulier (3 fois par an). ISSN : 1111-2050 (thèmes géographiques).

11 Journal algérien des régions arides.- Alger: Centre de recherches scientifiques et techniques sur les régions arides (CRSTRA), 2001- . Semestriel. ISSN : 1112-3273.

12 Revue de géographie et d'aménagement d'Oran.- Oran: Institut de géographie et d'aménagement, 1997- . Semestriel. ISSN : 1111-2174.

\section{Cameroun}

13 Héritages des tropiques: revue de recherches d'histoire et géographie.- Yaoundé: Université de Yaoundé, ENS, Département de géographie, 1997- . Semestriel. ISSN : 1029-872X.

14 Revue de géographie du Cameroun.- Yaoundé : Université de Yaoundé, 1980-1996. Semestriel; ISSN : 0254-3982.

\section{Cote d'Ivoire}

Annales de l' Université d' Abidjan, série G géographie.- Abidjan: Institut de géographie tropicale, 1969- 1984 . Annuel. ISSN : 0302-0924.

Bulletin d'information et de liaison des Instituts d'éthno-sociologie et de Géographie Tropicale.- Abidjan : Instituts d'éthno-sociologie et de Géographie Tropicale, 1967-1968. ISSN : 1013-6878. (devient Bulletin des Instituts de recherche de l' Université d'Abidjan , 1968-1969. ISSN : 1013-6886).

\section{Égypte}

17 Bulletin de la Société royale de géographie d'Égypte (titre abrégé de bulletin de la Société de géographie d'Égypte).- 1876-1991. Annuel. ISSN : 1110-5232.

\section{Ethiopie}

Ethiopian geographical journal.- Addis -Abeba: Mapping and geography Institute, 1963-1967. Irrégulier. 


\section{Ghana}

Bulletin of the Ghana Geographical Association .- Accra: Geographical Association, 1968-1975.

\section{Madagascar}

Madagascar: revue de géographie / Association des géographes de Madagascar; Université de Madagascar, laboratoire de géographie.- Tananarive: Université de Madagascar, 1962- . Semestriel. ISSN : 0047-5416.

21 Tsingy: revue de l'Association des professeurs d'histoire et de géographie.Tananarive : Association des professeurs d'histoire et de géographie, 2005- . Irrégulier. ISSN : 1998-9784.

\section{Maroc}

Annales du Service de physique du globe et de météorologie de l'Institut scientifique chérifien.- Rabat: Ministère de l'éducation nationale et des sports / éditions Félix Marcho, 1934-1963. Annuel. ISSN : 0221-3953.

Bulletin du Groupe de Recherches sur le Rif : études spatiales.- Tétouan: Groupe de Recherches sur le Rif, 2000- . Irrégulier.

Revue de géographie du Maroc.- Rabat: Société de géographie du Maroc, 1962- . Annuel. ISSN : 0035-1156 (suite de Revue de géographie marocaine, suite de Bulletin de la Société de géographie du Maroc, suite de Notes marocaines).

Travaux de l'Institut Scientifique Chérifien: série géologie, géographie physique.Rabat : Éditions marocaines et internationales, 1951-1968. Irrégulier. ISSN : 0851-299X (suite de Travaux de l'Institut scientifique Chérifien).

\section{Mozambique}

Boletim de la Sociedad de Geografia de Moçambique.- s.l : s.n., 1881- . ISSN : 2073-7718. Memorias de Instituto de investigaçao cientifica de Moçambique, ser. B sciencias geograficas e geologicas-. Lourenço Marques: Instituto de investigaçao cientifica de Moçambique, 1965- . Irrégulier. ISSN : 007661176.

\section{Nigeria}

Nigerian geographical journal.- Ibadan: Geographical Association, 1957-2000. Trimestriel. ISSN : 0029-0084.

\section{Sénégal}

Bulletin de l'Institut fondamental d'Afrique noire, série A sciences naturelles.- Dakar : IFAN, 1966- . Trimestriel. ISSN : 0018-9634 (suite de série A sciences de la vie et de la terre). 
Notes de biogéogaphie.- Dakar: Université cheikh Anta-Diop, laboratoire de biogéographie, 1986-1989. Annuel.

\section{Tunisie}

Publications de la Faculté des sciences humaines et sociales de Tunis.- Tunis : Faculté des sciences humaines et sociales, 1994-. Irrégulier. (suite des Publications de 1' université de Tunis, faculté des sciences humaines et sociales, 2éme série:géographie). Revue tunisienne de géographie.- Tunis: Université de Tunis, Faculté des lettres et sciences humaines, 1978- . Semestriel. ISSN : 0330-9924.

\section{Revues électroniques}

\section{Maghreb}

Bulletin des sciences géographiques : version électronique sur le site www.Inct.mdn.dz, 1998-2007, consultation en ligne des sommaires.

34 Géographie et aménagement: bulletin de l'association de géographie et d'aménagement du territoire/université Es-Senia d'Oran, Faculté des sciences de la terre, département de géographie et aménagement du territoire. www.agatoran.multimania.com

Journal algérien des régions arides/CRSTA.- Biskra: Centre de recherches scientifique et technique sur les régions arides. (information par mail : crstra@netcourrier.com)

Revue des sciences agronomiques et forestières.- Batna: Université Colonel Hadj, Institut d'agronomie. www.univ-batna.dz

37 Revue des énergies renouvelables. Alger: Centre de développement des énergies renouvelables. www.cder.dz

Revue maghrébine d'urbanisme et aménagement.- Alger: EPAU; Rabat: INAU. www.urbamag.nd

\section{Madagascar}

Mada-geo journal des sciences de la terre.- 1, 1998-14, 2009. http:// madarevues.recherches.gov.mg

\section{Mali}

Recherches africaines: annales de la faculté des lettres et sciences humaines, sous comité géographie-démographie. www.recherches-africaines.net /

\section{Namibie}

41 Namibian developement journal/ university of Namibia. http://ndj.unam.na/ 